\title{
The Agent based Scada Control Systems
}

\author{
Ahmad Almadhor \\ Aljouf University (Saudi Arabia)
}

\begin{abstract}
Supervisory control and data acquisition (SCADA) is a PC or computer system for gathering, dissecting and checking continuous and real time data. SCADA systems are utilized to monitor and control plants or equipments in a mixed bag of present day commercial enterprises. SCADA is a critical information system; its criticality originates from the way that SCADA systems are at present crucial parts of most countries' critical infrastructures; additionally systems may bring about cataclysmic results. SCADA as a critical information system confronts the same difficulties of present and future data information systems, for example, dynamicity and openness of work spaces, productivity, intricacy, and dependability/reliability, and so on. This research article depicts how Software Agents development can be brought into play to improve or perk up the unflinching nature or reliability of a control system.
\end{abstract}

\section{Keywords}

SCADA, Reliability, Software-Agent Technology

\section{INTRODUCTION}

In the latest couple of years, distributed computation has been the subject of various propositions and research exploration [J. Jing et al][ H. Nwana et al][ T.J Hess et al][ A. Bieszczad et al] [M. Satyanarayanan]. The energy for such area has achieved different upgrades in networking, inciting the improvement of internetworking protocols and the arrangement of a broad geographic network

so called Internet to complete them [A. Fuggetta et al] [A.Bieszczad et al]. We depict how the headway of a directory services protocol using Software Agent development extended the theoretical reliability of SCADA. Software agents are self-administering program units maintained by an execution setting; software agents can bring into play the network to send themselves to distinctive processors, in like manner moving among PCs. Furthermore, software agents can interact with each other on the network. This grants them to be utilized as fundamental building blocks for multifaceted systems. Control systems can benefit by agent technology from different perspectives. As an issue of first significance, disengagement or modularity is the key requisite of a control system and a key property of an agent system, so a control system can benefit by the adequately existing agent structures. Subsequently, self-principle or autonomy is another facet for agents that allow the customer to satisfy the endeavors in a group manner with the PC or computer. Along these lines, instead of honing complete control and expecting risk for every move the system makes, people can tune in a supportive process in which both individual and PC agents begin correspondence, screen events, and perform errands to meet a customer's targets. Third, through this technology experts take action and change their environment. Case in point, once an agent has completed its errand on a machine or is not ready to do it, the agent moves. The steadfastness or reliability of a SCADA system depends on upon its parts/components. It will be acknowledged that ' $n$ ' particular components are required to construct the system carry on working. Furthermore, every component's constancy can be seen as self-ruling of each other's. A regular enduring quality model for this sort of system is in light of the joint dissemination of $\mathrm{n}$ discretionary variables. Since they are self-ruling, their probability movement can be figured as the fundamental of the blend of the individual dissemination or distribution functions. In the most negligible case, one can expect a binomial distribution for the cumulative probability of collapse at any given time; thusly the probability of collapse will be higher than the probability of any one system missing the mark. This is a prompt determination of the way that the components are joined in plan or series. Furthermore, any reiteration can be deciphered in a parallel for each component, thus reducing the probability of breakdown for the component however shockingly any such overabundance must be close-by. So to make the full system monotonous or redundant it would be essential to twofold the components amount. Clearly, the ideal response for this issue would be a joker component, prepared for tolerating the sort of whatever other component, and thusly reasonably having the ability to supplant any of them. This would twofold the system reliability or steady quality with the base expense.

\section{INFRASTRUCTURE OF SCADA AND ITS EXECUTION ANALYSIS}

A customary SCADA system is based upon a social affair of utilitarian block that recognize committed handiness or functionality, for instance, data getting, decision reinforce, database updates or redesigns. This affirmation of a SCADA system encounters the insufficiencies presented in the past zone, as its unfaltering (reliability) is for the most part connected with the flourishing of every component it is comprised. In spite of the way that for low-level components like PLC, it might be possible to envision redundancies, it is extreme to supplant dedicated servers as every needs particular prescription.

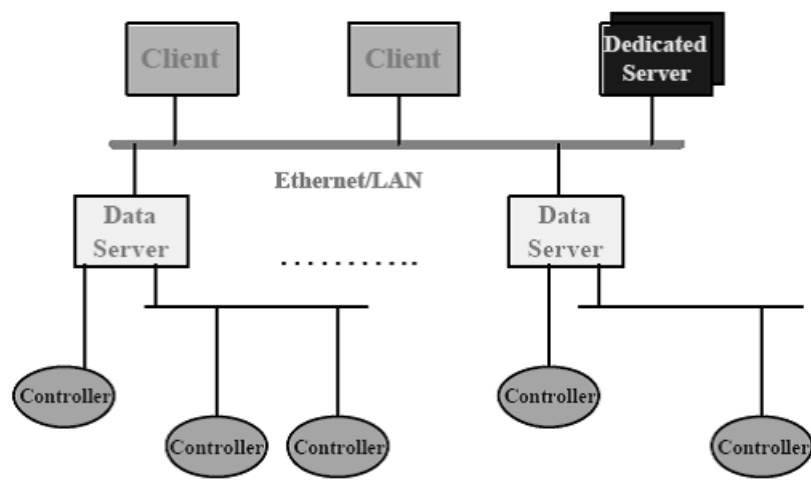

Fig 1: Typical SCADA Structural Design

Putting into practice or make the agent execution environment (AEE) functional to a consistent distributed control systems expects to develop another protocol for an appropriated control system that brings into play mobile agents. An agent execution environment is an software system that gives a runtime space to agents to execute, a standard interface for 
collaborations, services for formation, movement/migration and end of mobile agents, reinforces agents versatility and correspondence or communication while offering security to both agents and hosts. The essential inspiration driving the AEE is to host agents and grant them to run. Basically, an AEE is made out of two guideline sorts of agents: system agents and user defined agents. System agents are made by the AEE and are employed to facilitate the AEE work through the exercise of their services. On the other hand, user-defined one is agents which make use of the services of the AEE to work. A mobile agent system involves one of more AEE associated together. An agent execution environment will involve various services that energize and control mobile agents. The application of an agent based structure/system and structural design to a SCADA system is for the most part direct on an essential level. Regardless, some design issues need to be had a tendency to at indisputably the beginning stage of the depiction. As an issue of first significance, it is imperative to recognize the mixture purposes of the SCADA system architecture, given an agent based environment. There are three essential parts of an agent based system that are productive for a SCADA system: communication, mobility, and versatility. The directory service system protocol is basically used to discover agents in the associated agent execution environments, or to give direction for the present agents to diverse agents. There are two sorts of directory service: the local director service agents and the central directory service agent. Each directory service is given an exceptional name in the agent execution environment in the midst of its initialization. These sureties' territory apparent names at the application level. In this way, every service makes out how to find the directory service itself. On the other hand, agents must be perceived uncommonly in the milieu in which they work. Fitting recognizing confirmation licenses control, correspondence/communication, coordinated effort, and coordination of agents to happen.

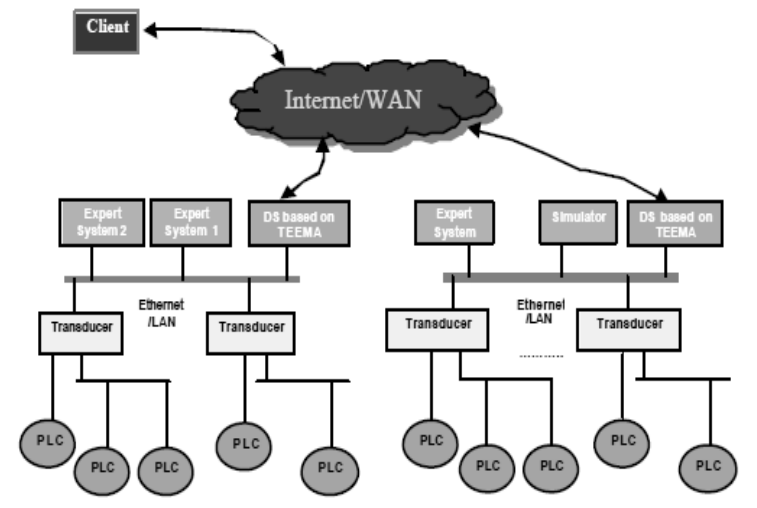

Fig 2: Agent Based SCADA

A SCADA system take benefits of various aspects gave by an AEE. Above all, the most crucial component is territory independence. With it, an agent is not any more limited on a singular machine, notwithstanding it can be gotten to wherever it is found. This makes it possible to execute a protected framework/approach, in which different copies of an agent may be started in particular territories or location, thusly reasonably improving the trustworthiness of the system. Another basic purpose of enthusiasm of agent systems is platform autonomy. While this tries a cost (generally speed) it is of exceptional help for components that now can take a shot at any workstation equipped with the AEE. The AEE thus makes the workstation aware of distinctive workstations, thusly understanding a more refined sort of a network or in other words "an awareness cluster".

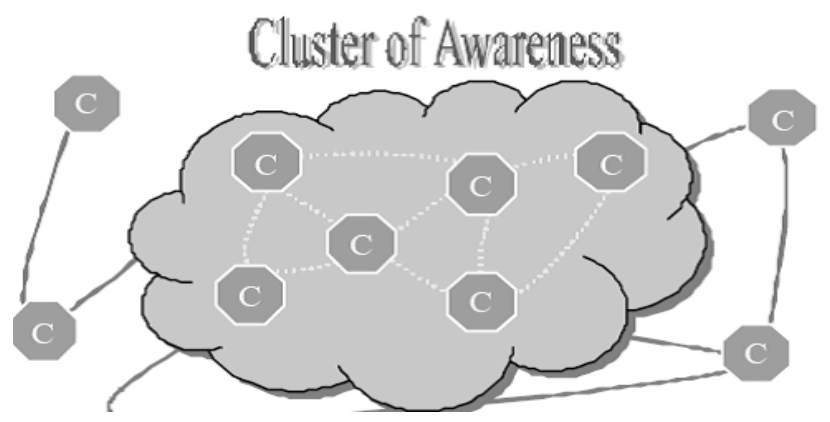

Fig 3: Cluster of Awareness

An AEE changes the fundamental structure of the SCADA system, yet no it's theoretical structural design. Really, the determination of an AEE streamlines the generation of modules and advances a distributed model without having the need to take off upgrades to the SCADA structural design itself. Regardless, since the agent's standard obliges extensive changes in the undertaking code, it can be lavish to increase a SCADA system for software agents, and it is also not feasible to change the entire structure overnight. Transformative development or migration patterns can be used that change the current SCADA system into an agent system by logically supplanting components with their agent's accomplices, while meanwhile giving stub interfaces to the non-agent components.

\subsection{Execution Analysis}

The most basic bit of an agent system that executes a directory service is, clearly the directory itself. The work outlined here has been putted into practice with the TEEMA execution environment. TEEMA shorts for TRLabs Execution Environment for Software Agents. TEEMA is a lightweight development environment made at TRLabs, a western Canadian affiliation taking in western Canadian regional governments, schools, and associations. TRLabs' goal is to develop precompetitive investigation endeavors, and TEEMA identifies with one such result. TEEMA was collected or constructed for fast agent operation, with the objective researchers ought to study the properties of agent based programming without being obliged by code distance, as is consistently the case for business systems. TEEMA is particularly segregated, and likewise it is greatly changeresistant. Notwithstanding the way that TEEMA has not been attempted in mechanical circumstances, regardless any result conceivable in such a bare bones milieu can be copied smoothly in a more generous system. The TEEMA directory service is an agent itself and in this manner it has no excellent advantages, other than the probability to show its name. The directory services passes on to distinctive subsystems utilizing a standard protocol. The model for the agent is that of an event driven agent with storage. This allows the agents to bestow a service that can either keep up a state or not. It is basic to notice that this agent does not have any reference to the protocol that it makes use of to talk with the agent requesting its services; it must come, then again, with specific prerequisites when taking up with the directory service. Each directory service agent keeps up three gatherings. The main stays educated concerning the service agent's name and address. The second one tracks non-neighborhood services names and areas. The last one is simply kept up by the central directory service: it is a Directory Service Name table that is made out of the directory service name and the address of 
directory service agent, to discover where the other director services stay. If all else fails, each AEE should have one or more than one agent running meanwhile and each agent is by and large designated specific assignments to wrap up. Agents can all be aware of and talk with each other, despite when they are in particular machines. The directory service system is a service bestowed by agent, running as a system agent. All users define agents that are brought into play as a piece of an AEE are subclasses of the base agent class and are started by the customer. The directory service system in a AEE should give the going hand in hand with facilities or services [Andrian Gligora et al]:

\section{Directory service agent formation}

The registry service agent is running as a bit of the system agent pool. It should be made when an AEE starts. It is now on the customer to start it as The Local Directory Service Agent or The Central Directory Service Agent.

\section{Sign in}

Every service agent that is made in an AEE will select itself to the directory service agent with two parameters: service agent name and service agent address. The director service agent furthermore signs into the central directory service agent for whom the trade happens between the local directory service agent and the central directory service agent just for the sake of zone flexibility.

\section{Sign off}

Services must enroll with the director service when they are made, yet they ought to similarly tell the directory server when they wish to shut down. This is done to redesign the table of services for each agent platform; really, it may happen that a bit of the agents experience issues and as needs be they are not ready to respond. This is implemented through the use of probe or query messages. In the occasion that messages can't be passed on, then the services get the chance to be occupied, and automatic close down (sign-off) is approved. In the occasion that messages can be passed on, a long timeout is well thought-out. In decisively the same way, the directory service shuts down or sign-off from the central directory service when it is quit running the system.

\section{Query}

Each agent can scrutinize the directory service for other service agent in both the same and unmistakable ranges. To begin with, the directory service agents query database to make sense of whether a requested service is in its neighborhood surroundings or not. In case it can't avoid being, it will give the result to the requesting agent. Of course, if the requested service is not in the area environment, the directory service will forward the request to the central directory service agents and sit tight for the result within a predefined measure of time or long timeout. The central directory agent can demonstrate a request to other adjacent directory services, once it gets an inquiry from another directory service agent and can't find it in its environment. The central directory service agent will in like manner demonstrate the requests to other list service agents if its table does not contain a service being inquired.

\section{Cache}

Each trade in an AEE will be kept in the tracking collection. A valid example, if an agent requests a service which it is not in its adjacent surroundings, the area directory service will request the central directory service and when it gets the opportunity to be known where the requested agent is, the close-by directory service will keep the agent name and its address into the collection. If the same service is asked to the directory service afresh, the directory service can think its region from the track table particularly. It is moreover basic to update these records oftentimes. It is possible that a specific service migrates starting with one platform then onto the next, yet this is managed by the AEE. Maybe, if a service agent stops to exist in a non-smooth way, it is fundamental to have a recovery approach to ensure reliability of the table. An examination of these systems yields that there are two sorts of channel that are brought into play to talk within the settings with the directory service system: the Internal Channel and the External Channel. The Internal Channel is used for any trade among agents and the directory service in AEE For example, after the directory service agent gets a requesting, it doubts whether the requested agent is in its surroundings or not. If it is, the directory service agent sends a results message to the requesting agent. A while later, the two agents have the ability to relate with each other. The External Channel is brought into play as a piece of solicitation to pass on among directory service agent. From the above delineation, the directory service agent, exercising within channel, goes about as the Close-by directory service agent. If it can't find the requested agent in its surroundings, it propels the request to the central directory service employing the external channel, and subsequently it sits tight for a response within a certain measure of time.

\subsubsection{Merits}

There are various central purposes of applying the agent development to a distributed control system, as takes after:

\section{a) Reliability or steadfastness}

Steadfast quality or reliability is the vital concern here, and since the AEE can change a strong SCADA system into a parallel system, the trustworthiness of a agent-based SCADA is exceptionally overhauled. In addition, it is possible to have an unfaltering quality service whose sole undertaking is to screen the present services and make them open to diverse parts of the SCADA system. This system recovery device makes the system more fault-tolerant.

\section{b) Adaptability or Versatility}

Mobile agent's usage over the Internet upgrades the SCADA system versatility. Not simply every system is consistent and fault-tolerant in their site, then again they are moreover prepared to get to other remote systems if the need come to pass.

\section{c) Integration}

A SCADA system executing an agent structural design is a great deal less requesting to arrange than their non-agents accomplice. This fundamental result gets particularly from the qualities of the agents architecture. Agents are inalienably specific and they give through a settled approach. Thusly, they can be executed in an unmistakable way, and their structure can end up being duller, which advances reuse and superiority read-through.

\section{d) Remote access}

The network infrastructure and the unavoidable flowed nature of the agent structural design make it possible to control the SCADA system remotely, for case; managers can control their plants from remote destinations by means of the Internet.

\subsubsection{De-merits}

Clearly, every system building effort needs to consider tradeoffs and potential shortcomings, and the structure delineated here is no exception. Different dilemmas must be 
well thought-out prior to grasping agent development for SCADA systems. These dilemmas are the going with:

\section{a) Availability of network}

Regardless of the way that the SCADA system is facilitated and reliable, and thusly it can abuse the remote execution of their agent errands in distinctive systems, it is still dependent on the openness (availability) of the Internet or other network affiliation. A valid example, if the site page can't join with the Internet or to another framework/network, the probability to handle the unflinching quality issue by employing mobile agent is compelled to the local system. If the local system is also not any more prepared to support a network, then the whole structural design gets the opportunity to be insufficient. Thusly, it is discriminating to have a strong framework/network environment. This however is ending up being more a need in any business environment, and it doesn't influence particularly much on the site outlays.

\section{b) Protection or Security}

At whatever point a system exercise a network, potential security dangers develop. They stretch out from clear network downtime dilemmas to more many-sided interferences, especially when the system brings the internet into play as a system for correspondence/communication between remote territories. Various courses of action have been proposed for this concern. One incredible catch-all game plan is to secure the network by strategy for either a private or a virtual private network system. This will deflect message "snooping" or more repulsive, changing, from unapproved individual. At present, TEEMA fuses a security communication system based on the Java Secure Sockets Extensions. This system can be further strengthened with the gathering of hardware-based security courses of action. The Java Secure Sockets Extension system as well gives an instrument to secure unmistakable proof of the passing on associates. This makes it possible to predestine a game plan of trusted machines that can take an enthusiasm for the control system and maintain a strategic distance from interferences. Finally, internal agent's environment security must be well thought-out. As the system stands now, there is the strong believability that a agent crashes an entirety machine. To avoid this, it is vital to complete a wellbeing part for agents, ideally in perspective of the obtainment of levels of execution that make it attainable for a more attempted agent to run closer to the system, however a less attempted system won't be allowed to bring into play system calls.

\section{c) Constancy or Dependability}

In spite of the way that the steadfastness of the system grows in wording of adjustment to non-discriminating disappointment and insufficiency recovery, it is essential to reflect on the way that another system is at present in plan with the SCADA system, the network connection. The unflinching quality (reliability) examination ought to now mull over a substitute circumstance. The trade off brought in by this novel arrangement is tolerably straightforward to concentrate, notwithstanding it depends on upon the availability of the network structure and the probability to make it as tried and true as desirable. This could moreover entail the usage of unprecedented network hardware, for instance, recoverable connection gateways, and must be joined in the fiscal examination for the allocation of such a system. At present, the availability of network does not give off an impression of being a matter and most SCADA systems are currently interacting with the basic control line via the network.

\section{CONCLUSIONS}

The directory service is brought into play fundamentally to furnish or bestow the subsystem the aptitude to discover where services of other subsystem are placed. This philosophy is more integrated, adaptable/flexible, multitalented, and consistent than the customary strategy. Moreover, the aforementioned structural design fuses the network structure in the SCADA system, making it suitable for remote screening or monitoring and control applications. The system also presents a couple detriments, fundamentally on account of the new flowed structural design (architecture). Prospect and potential exertion can lend a hand to vanquish these insults. The distinctively flowed or distributed structure of the agent environment gives an expects to zone opportunity or independence, yet the method for the directory service obliges that specific names be affixed to each system. In the present use, there must be a central directory service to which each adjacent directory system must schedule. Future executions will execute a discovery service that allows dynamic revelation of directory services with an instrument like ARP address discovery. Notwithstanding the way that agents can keep running in every practical sense on any platform, there is still the need to have the same AEE on every system. This can transform into an inconvenience if agents need to be joined with submitted circumstances running specific AEE. This can come about for a combination of reasons, however for the most part it is a matter of security, for example, it might be that wireless communication suppliers trust only their specific agent platforms. This can be overcome by means of automatic translating systems that will allow agents semantic uniformity in both platforms. This thought is being clarified in another casing by Web engineers with new-fangled structural designs that get the opportunity to be free from the platform or zone. They may possibly be well thought-out for the Internet applications as a development of Object Oriented Programming (OOP's).

\section{REFERENCES}

[1] "A. Bieszczad et al...", "Mobile Agents For Network Management", Carleton University, IEEE Communications Surveys, Volume: 1; No. 1; Fourth Quarter 1998. IEEE Press

[2] "A. Fuggetta et al...". "Understanding Code Mobility", IEEE Transactions on Software Engineering; Volume: 24; No. 5; May 1998. IEEE Press.

[3] "T.J Hess et al..." "Using autonomous software agents to create next generation of decision support system". Decision Sciences; Atlanta; Winter 2000

[4] "J. Jing et al..." "Client-server computing in mobile environments", ACM Computing Surveys. Volume: 31 ; Pages 118-157; Baltimore; Jun. 1999. ACM Press.

[5] "M. Satyanarayanan", "Accessing information on demand at any location Mobile Information Access", IEEE Personal Communications, Feb. 1996. IEEE Press.

[6] "H. Nwana et al..". "An Introduction to Agent Technology", Intelligent Systems Research Applied Research and Technology, BT Labs.

[7] "Andrian Gligora et al...Development of a service oriented SCADA system, Procedia Economics and Finance 3 ( 2012 ) 256 - 261, published by Elsevier 